\title{
Traumatic event exposure associated with increased food insecurity and eating disorder pathology
}

\author{
Carolyn Black Becker ${ }^{1, *}$, Keesha Middlemass ${ }^{2}$, Clara Johnson ${ }^{1}$, Brigitte Taylor ${ }^{1}$, \\ Francesca Gomez ${ }^{1}$ and Autumn Sutherland ${ }^{1}$ \\ 'Department of Psychology, Trinity University, One Trinity Place, San Antonio, TX 78212-7200, USA: \\ ${ }^{2}$ Department of Political Science, Trinity University, San Antonio, TX, USA
}

Submitted 31 December 2017: Final revision received 6 April 2018: Accepted 29 May 2018: First published online 15 August 2018

\begin{abstract}
Objective: The primary aim was to investigate the association between food insecurity (FI) and eating disorders, which are nutrition-based public health problems, with traumatic event exposure in a low-income marginalized population. The study also investigated the association between traumatic event exposure, anxiety and weight stigma.

Design: The study used self-report surveys in a cross-sectional design.

Setting: Food pantries affiliated with the local food bank in a major US city.

Subjects: Participants ( $n$ 503) consisted of clients presenting to food pantries. Participants were predominantly female (76.5\%), Latino/Hispanic (64.6\%) and low-income (59\% reported earning under \$US 10000 per year).

Results: Results indicated that $55.7 \%$ of participants had directly experienced a traumatic event; this increased to $61.6 \%$ when witnessing was included. Higher levels of FI were associated with greater traumatic event exposure. Increased exposure to traumatic events correlated with worsened overall eating disorder pathology $(r=-0 \cdot 239)$, weight stigma $(r=-0 \cdot 151)$ and anxiety $(r=-0 \cdot 210)$.

Conclusions: The present study is the first to investigate the association of FI, eating disorders and trauma in a low-income marginalized population. Results indicate that exposure to traumatic events is common in this civilian population and that traumatic event exposure is associated with higher levels of FI and eating disorder pathology. Results indicate that further research is warranted given that traumatic event exposure, eating disorder pathology, weight stigma and anxiety may complicate effective delivery of public health interventions in those living with FI.
\end{abstract}

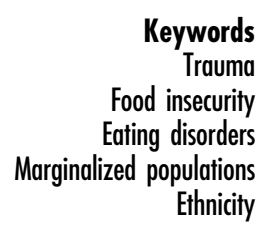

Food security signifies when people have sufficient access to appropriate, nutritious and safe food that allows them to both meet dietary needs and live active and healthy lives ${ }^{(1,2)}$. In contrast, food insecurity (FI) commonly refers to a state in which individuals and/or families lack sufficient access to safe and nutritious food as a result of inadequate resources ${ }^{(3)}$. FI is not only common in low- and middle-income countries, but also impacts those living in high-income countries, such as the USA. Indeed, the US Department of Agriculture estimates that almost 49 million people qualified as foodinsecure in $2015^{(3)}$. FI varies in severity and the people who are affected; it can range from individual adults being unable to access a sufficiently varied diet that meets nutritional needs to having hungry children in the household. Importantly, reports of hungry children present in the household typically are presumed to indicate the presence of even hungrier adults, given the propensity for most caregivers to prioritize food allocation to children ${ }^{(4)}$.

FI is a significant public health problem. For instance, research supports a strong association between FI and diabetes $^{(5)}$. Research also links FI with CVD risk factors such as hypertension and hyperlipidaemia ${ }^{(6)}$, as well as self-reported CVD and food allergies ${ }^{(7)}$. Further, research indicates that FI may confer additional risk for chronic disease above and beyond other factors, such as poverty. More specifically, research indicates that those who are low-income and living with FI have a lower Healthy Eating Index-2005 score relative to those who are low-income and food-secure ${ }^{(8)}$. In addition, even when macronutrient content is similar, low-income FI is associated with the type of poor diet quality that increases risk for chronic disease ${ }^{(8)}$. 
In contrast to an extensive literature documenting the association of FI with physical health conditions such as diabetes, comparatively few studies have explored FI and mental health outcomes that are considered public health problems. To date, most of the latter studies have focused on depression/dysthymia, anxiety disorders and/or more broadly defined common mental disorders ${ }^{(7,9-13)}$; one recent study, however, also found that higher levels of FI were associated with increased rates of clinically significant eating disorder (ED) pathology ${ }^{(14)}$. More specifically, among those who met criteria for the most severe level of FI, a category which included adults who reported having hungry children at home, $17 \%$ met criteria for a clinically significant ED. Regarding specific ED behaviours for adults in the child hunger group, the most severe form of FI, $37.6 \%$ reported binge eating or objective overeating, $38.3 \%$ reported night eating with distress, $20 \cdot 4 \%$ reported vomiting after eating to control weight and shape, and $22.8 \%$ reported using laxatives or diuretics to control weight and shape.

The findings from Becker et al. ${ }^{(14)}$ are concerning for several reasons. First, these rates are substantially higher in than nationally representative samples. For instance, Hudson et al. ${ }^{(15)}$ found a 12 -month prevalence rate of $7 \%$ for any ED (including sub-threshold binge eating disorder) in the National Comorbidity Sample; this is substantially lower than the $17 \%$ of child hunger food-insecure adults meeting current criteria for an ED in Becker et al. ${ }^{(14)}$. In addition, the 12-month prevalence for any binge eating in Hudson et $a l{ }^{(15)}$ (5.7\%) was substantially lower than the one-month prevalence of any binge eating in Becker et al. (i.e. objective and subjective combined $=25.7 \%$ ). Second, ED have been identified as a significant public health concern in and of themselves, leading to at least some role impairment ${ }^{(15)}$ and comparable impairment in quality of life to depression and bipolar disorder ${ }^{(16)}$. Third, ED pathology, by definition, indicates that individuals struggle to manage their eating behaviour. As such, it may impede efforts in food-insecure populations to change eating behaviours aimed at impacting physical health conditions (e.g. diabetes) identified as significant public health concerns. This is particularly likely if the ED is unreported, which is common. Finally, ED are psychologically distressing for both the individual and family members who are aware of the ED; they also may create additional stress for a food-insecure household if out-of-control binge eating impedes family attempts to stretch limited food resources from pay cheque to pay cheque.

Importantly, increased FI in the Becker et al. ${ }^{(14)}$ study also was associated with increased internalized weight stigma (i.e. biased and prejudicial attitudes towards those with heavier bodies) and anxiety. Internalized weight stigma has been identified as potentially increasing the likelihood of metabolic syndrome ${ }^{(17)}$. In addition, when present, both internalized weight stigma and anxiety will likely complicate public health efforts to address food and physical health concerns among those with FI. As such, the present study highlights the need to better understand other variables that may interact with ED pathology, weight stigma and anxiety in those living with FI, such as trauma exposure.

To date, limited research has investigated the association of FI and trauma exposure. This is surprising given that: (i) maternal mental health has been proposed as an additional risk factor for household FI beyond low socio-economic status ${ }^{(18)}$; and (ii) trauma exposure has long been recognized as a non-specific risk factor for mental health problems ${ }^{(19,20)}$. Among the FI studies that have investigated trauma exposure, most have focused specifically on violence or sexual assault. For instance, Chilton et al. ${ }^{(21)}$ investigated exposure to violence and FI in forty women receiving public assistance. Results indicated that a greater percentage of women living with the highest level of FI reported exposure to violence compared with women living with less severe FI or who were food-secure. Similarly, Melchior et al. ${ }^{(18)}$ found that families with low socio-economic status who were foodsecure reported less maternal exposure to domestic violence than families with low socio-economic status who lived with FI. Finally, Wehler et al. ${ }^{(22)}$ found that women who reported experiencing childhood sexual assault were four times more likely to meet criteria for adult hunger in their households $v$. women with no childhood sexual assault history. In one of the few studies to investigate trauma exposure and FI more broadly, Roberts et al. ${ }^{(23)}$ examined exposure to an array of traumatic events among internally displaced persons in northern Uganda. Most, although not all, participants had experienced FI. Unsurprisingly, rates of trauma exposure were extremely high in this population (e.g. murder of a family member/friend $=74.8 \%$ ). To our knowledge, however, no study has investigated the prevalence of a wide array of potentially traumatic events and mental health in those living with FI in the USA.

The present study had four aims. First, we sought to document the prevalence of self-reported traumatic events in a low socio-economic, predominantly ethnic minority population visiting food pantries in a major US city. Second, we tested the hypothesis that those living with higher levels of FI would report greater traumatic event exposure. Third, because trauma exposure is considered a non-specific risk factor in that it increases risk for a range of mental health concerns, including $\mathrm{ED}^{(19)}$, we hypothesized that increased past exposure to traumatic events would correlate with greater levels of ED pathology, weight stigma and anxiety in our sample population. Finally, we hypothesized that past exposure to traumatic events would correlate with increased rates of specific ED behaviours, including frequency of objective binge eating, night eating with distress, vomiting and laxative/ diuretic use. 


\section{Methods}

\section{Participants}

Participants included 503 adult clients of food pantries that partner with the San Antonio Food Bank, Texas, USA. Most participants ( $72.8 \%$ ) reported being between 25 and 65 years of age $(25-50$ years $=39 \cdot 4 \% ; 51-65$ years $=33.4$ $\%)$ and identified predominantly as female (76.5\%). The majority of participants self-identified as Latino/Hispanic (64.6\%) and 16.5\% identified as Black/African American; $11.3 \%$ as White; $1.4 \%$ as Native American; and the remaining $4.4 \%$ as Other. Almost $60 \%$ of participants reported annual household income as less than \$US 10 000; only 3\% reported earning more than \$US 40000 per year. Thus, the sample was impoverished. Detailed demographics for the full sample broken down by level of FI are presented in Becker et al. ${ }^{(14)}$. In exchange for completing a questionnaire, participants received a \$US 5 gift card to a local grocery store chain.

\section{Procedure}

The present study was run in collaboration with the San Antonio Food Bank. After approving the study design, San Antonio Food Bank provided the locations and operating times of their partner food pantries. Research assistants then invited individual food pantries to participate. Procedure varied slightly based on the recommendations of pantry staff so as to not disrupt food distribution (see Becker et $a l^{(14)}$ for additional procedure details). Most often, food pantry staff introduced research assistants to clients while they waited to collect food. Upon introduction, research assistants then recruited clients using a standardized script. All materials and scripts were available in Spanish for non-English speakers. Before proceeding with the questionnaire, research assistants reviewed each component of the consent form using a second script, and then gave participants sufficient time to read the consent form and provide written consent. While participants completed questionnaires, research assistants remained nearby to address questions regarding comprehension or to assist participants who had difficulty reading. After questionnaire completion, research assistants debriefed participants, noted any participant feedback, provided participants with a list of local free or low-cost mental health resources, and then presented participants with their \$US 5 gift card.

\section{Measures}

In addition to providing demographic information, participants completed the Radimer/Cornell Food Insecurity Measure $\left(\right.$ RCFIM $^{(24,25)}$ ), which assesses FI over the past year. The RCFIM measures the continuum of FI; based on the RCFIM, participants were categorized as: not foodinsecure (i.e. do not meet clinical criteria for FI); household FI (i.e. experience anxiety about food, lack of dietary variety because of a lack of resources and food running out before the end of the month); individual FI (i.e. adults experience hunger at times because they lack food); and child hunger FI (i.e. adults experience inability to feed their children secondary to a lack of resources and are presumed to be even more hungry than those who are individual FI). Items for the RCFIM measures were rated on a Likert scale $(0=$ 'not true'; $1=$ 'sometimes true'; $2=$ 'always true'). Research supports the construct and criterion-related validity and the internal consistency of the RCFIM $^{(24)}$. Internal consistency within the present sample was excellent (Cronbach's $\alpha=0.941$; see Becker et $a l .{ }^{(14)}$ for additional detail on scoring of the RCFIM).

The Life Events Checklist (LEC) was used to assess exposure to potentially traumatic experiences from childhood to present. Developed by the National Center for Posttraumatic Stress Disorder, the seventeen-item LEC-5 has demonstrated good convergence with the Traumatic Life Events Questionnaire and acceptable temporal stability ${ }^{(26)}$. Scores on the LEC have been found to significantly correlate with post-traumatic stress disorder symptoms and measures of depression and anxiety ${ }^{(26)}$. The LEC is unusual in that it assesses different types of exposure to a wide variety of potentially traumatic events. Thus, the LEC may elicit information not gathered by measures that focus only on direct experience (e.g. structured interview or the Traumatic Stress Schedule, see Norris and Norris and Slone $\left.{ }^{(27,28)}\right)$. Given the limited information regarding trauma exposure in those living with FI in the USA, we selected it for its breadth of assessment.

For each type of potentially traumatic event, participants indicated whether they directly experienced the event, witnessed the event, learned about the event, are not sure about exposure or never experienced the event (i.e. does not apply). Direct exposure is given a score of 1 , witnessing $=2$, learning $=3$, not sure $=4$ and never experienced $=5$; thus, lower scores indicate more direct exposure to different types of traumatic events. To facilitate comprehension in a population with low education levels, we made minor alterations to some wording (e.g. replaced 'toxic substance' with 'poison') and added examples for some questions (e.g. adding 'lead, carbon monoxide, asbestos and radon' to the original example of 'dangerous chemicals, radiation').

Eighteen items from the self-report Eating Disorder Diagnostic Scale for DSM-5 (EDDS-5) assessed the spectrum of ED pathology over the past month ${ }^{(29)}$. The EDDS-5 is a slightly modified version of the EDDS-4, which was designed to address diagnostic criteria changes in DSM-5 (the Diagnostic and Statistical Manual of Mental Disorders, 5th edition). Research with the EDDS-4 supports the internal consistency, convergent validity with ED risk factors, criterion validity with interview-based diagnoses, sensitivity to change and predictive validity ${ }^{(29)}$. We selected the EDDS-5 for two primary reasons. First, based on extensive experience working with low-income 
and marginalized populations, one of the principal investigators judged the EDDS- 5 as somewhat easier to understand. Second, the EDDS-5 includes questions assessing night eating, whereas the EDDS-4 does not. Internal consistency for the EDDS-5 items was very good $(\alpha=0.911)$ in this sample. Per Stice et al. ${ }^{(29)}$ and Krabbenborg et al. ${ }^{(30)}$, we computed a standardized summed composite score in addition to calculating frequencies of specific behaviours (see Becker et al $^{(14)}$ for additional details on EDDS scoring and results).

Ten items from the Weight Self-Stigma Questionnaire (WSSQ) assessed the degree to which participants had internalized negative societal attitudes about heavier weight bodies (i.e. prejudice against individuals affected by being overweight or obese); for the present analyses, we used a summed total score ${ }^{(31)}$. We cut two items from the original measure because the research team judged the questions as too complex for a population likely to have low reading and comprehension levels. Research supports both reliability and validity for the WSSQ ${ }^{(31)}$. Participants are asked to indicate the degree to which they agree or disagree with a series of statements such as 'I don't have enough self-control to be thin'; there is no time frame in the instructions. Items are rated on a 7-point Likert scale ( $1=$ 'strongly disagree' to $7=$ 'strongly agree'). Internal consistency for the ten items was excellent $(\alpha=0.956)$.

We summed eight items from the well-validated Penn State Worry Questionnaire (PSWQ) to assess anxiety and worry ${ }^{(32)}$. We excluded items that were (i) deemed too complicated for individuals with a low reading level or (ii) reverse scored, which also raised issues about comprehension. Participants are asked to rate to what degree statements such as 'My worries overwhelm me' are typical; as with the WSSQ, no time frame is provided. PSWQ items used in the present study had excellent internal consistency in our sample $(\alpha=0.952)$.

Due to the low socio-economic status of the sample population, and because very low-income individuals from marginalized populations typically lack regular access to physicians, nurses and scales, we omitted questions about weight and height from the EDDS. Further, because many of those living with FI may have lower levels of education, all items were reviewed using online software (i.e. Microsoft ${ }^{\circledR}$ Word proofing tool which provides a Flesch-Kincaid Grade Level) and adjusted, as necessary, to a 6th grade reading level. As noted above, we removed more complicated questions; as such, only a few alterations were needed to adjust the reading level after review with the online software. Consequent to the prevalence of Spanish speakers in San Antonio, all measures were translated into Spanish by a bilingual member of the research team and then translated back to English by an independent bilingual consultant; back translation helps to ensure the meaning of questions remain equivalent in the two languages. Finally, a third bilingual person who grew up in San Antonio reviewed all items and made minor adjustments to reflect the Spanish dialect that is common in San Antonio (see Becker et al. ${ }^{(14)}$ for description of pilot testing for all measures).

\section{Data analysis}

To document the prevalence of self-reported traumatic events in this sample (Aim 1), we provide descriptive data (i.e. frequencies) for the total sample and break data down by self-reported gender, race/ethnicity and level of FI. We chose this approach because those living with FI are a marginalized population and the present study is the first we are aware of to investigate a wide range of trauma exposure in this population in a high-income country. Frequencies typically are easier to comprehend than means and are important to demonstrate the degree to which a given problem is normative enough to be viewed as a possible public health problem ${ }^{(33)}$. To test the degree to which trauma exposure worsened as FI increased (Aim 2), we conducted one-way ANOVA. For our final two aims, we hypothesized that scores on the LEC would correlate with ED pathology, weight stigma, anxiety (Aim 3) as well as frequencies of specific ED behaviours (Aim 4). Because greater trauma exposure yields a lower LEC score, we expected negative correlations which would indicate that increase trauma exposure was associated with increased ED pathology, weight stigma and anxiety. For these aims we ran bivariate correlations. We used a $P$ value of $<0.05$ to indicate statistical significance.

\section{Results}

Based on results from the RCFIM, the sample was divided into four groups: not food-insecure ( $n$ 41), household FI ( $n$ 45), individual FI ( $n$ 190) and child hunger FI ( $n$ 227); child hunger represents the most severe level of FI. We describe the first group as not food-insecure, instead of food-secure, because this group went to food pantries to secure food; this behaviour suggests that they are not completely food-secure.

Overall, $55.7 \%$ of the sample reported directly experiencing at least one potentially traumatic event; this percentage increased to $61.6 \%$ when witnessing an event was included. Table 1 displays frequencies for the sixteen specific types of traumatic events assessed by the LEC for the total sample and broken down by gender and race/ ethnicity. Only direct experiences (i.e. 'happened to me') with traumatic events are listed in Table 1 . Because no participant reported directly experiencing an 'other' traumatic event (i.e. item 17), this question is not included in Table 1.

Exploratory $\chi^{2}$ analyses, with a Bonferroni correction setting $P<0.002$ for multiple unplanned comparisons, revealed only four group differences in rates of trauma exposure. We found a gender difference for both serious 
Table 1 Frequencies of potentially traumatic events among a sample of clients presenting to food pantries affiliated with the San Antonio Food Bank, Texas, USA, July-December 2016

\begin{tabular}{|c|c|c|c|c|c|c|c|c|c|c|c|c|}
\hline \multirow[b]{2}{*}{ Event } & \multicolumn{2}{|c|}{$\begin{array}{l}\text { Total sample } \\
\quad(n \text { 503) }\end{array}$} & \multicolumn{2}{|c|}{$\begin{array}{l}\text { Male } \\
(n 113)\end{array}$} & \multicolumn{2}{|c|}{$\begin{array}{l}\text { Female } \\
(n \text { 385) }\end{array}$} & \multicolumn{2}{|c|}{$\begin{array}{l}\text { African American } \\
(n \text { 83) }\end{array}$} & \multicolumn{2}{|c|}{$\begin{array}{l}\text { Latino } \\
(n 325)\end{array}$} & \multicolumn{2}{|c|}{$\begin{array}{l}\text { Other } \\
(n 89)\end{array}$} \\
\hline & $n$ & $\%$ & $n$ & $\%$ & $n$ & $\%$ & $n$ & $\%$ & $n$ & $\%$ & $n$ & $\%$ \\
\hline Natural disaster & 76 & $15 \cdot 1$ & 22 & $19 \cdot 3$ & 54 & 14.0 & 14 & $16 \cdot 9$ & 41 & $12 \cdot 6$ & 20 & 22.5 \\
\hline Fire or explosion & 55 & 10.9 & 18 & $15 \cdot 8$ & 37 & 9.6 & 14 & $16 \cdot 9$ & 31 & 9.5 & 10 & 11.2 \\
\hline Wreck, crash accident & 174 & 34.6 & 49 & 43.0 & 125 & 32.5 & 29 & 34.9 & 103 & 31.7 & 40 & 44.9 \\
\hline Serious accident & 107 & 21.3 & 40 & $35 \cdot 1$ & 67 & $17 \cdot 4$ & 15 & $18 \cdot 1$ & 64 & 19.7 & 26 & 29.2 \\
\hline Poison exposure & 41 & 8.2 & 17 & 14.9 & 24 & $6 \cdot 2$ & 3 & 3.6 & 30 & $9 \cdot 2$ & 7 & 7.9 \\
\hline Assault with weapon & 84 & $16 \cdot 7$ & 30 & $26 \cdot 3$ & 54 & $14 \cdot 0$ & 12 & 14.5 & 53 & $16 \cdot 3$ & 18 & $20 \cdot 2$ \\
\hline Sexual assault & 90 & $17 \cdot 9$ & 12 & 10.5 & 78 & $20 \cdot 3$ & 11 & $13 \cdot 3$ & 50 & $15 \cdot 4$ & 28 & 31.5 \\
\hline $\begin{array}{l}\text { Other unwanted sexual } \\
\text { experience }\end{array}$ & 74 & $14 \cdot 7$ & 12 & $10 \cdot 5$ & 62 & $16 \cdot 1$ & 10 & 12 & 40 & $12 \cdot 3$ & 23 & $25 \cdot 8$ \\
\hline Physical assault & 119 & 23.7 & 29 & 25.4 & 90 & 23.4 & 15 & $18 \cdot 1$ & 67 & $20 \cdot 6$ & 36 & $40 \cdot 4$ \\
\hline Combat or war exposure & 32 & $6 \cdot 4$ & 19 & $16 \cdot 7$ & 13 & 3.4 & 8 & $9 \cdot 6$ & 17 & $5 \cdot 2$ & 7 & 7.9 \\
\hline Captivity & 48 & 9.5 & 13 & 11.4 & 35 & $9 \cdot 1$ & 5 & $6 \cdot 0$ & 30 & $9 \cdot 2$ & 12 & 13.5 \\
\hline Life-threatening IIIness or Injury & 97 & $19 \cdot 3$ & 32 & $28 \cdot 1$ & 65 & $16 \cdot 9$ & 13 & $15 \cdot 7$ & 56 & $17 \cdot 2$ & 26 & 29.2 \\
\hline Severe human suffering & 30 & $6 \cdot 0$ & 10 & $8 \cdot 8$ & 20 & $5 \cdot 2$ & 5 & $6 \cdot 0$ & 16 & 4.9 & 9 & $10 \cdot 1$ \\
\hline Sudden violent death & 39 & $7 \cdot 8$ & 11 & $9 \cdot 6$ & 28 & $7 \cdot 3$ & 6 & $7 \cdot 2$ & 23 & $7 \cdot 1$ & 10 & $11 \cdot 2$ \\
\hline Sudden accidental death & 42 & $8 \cdot 3$ & 7 & $6 \cdot 1$ & 35 & $9 \cdot 1$ & 6 & $7 \cdot 2$ & 27 & $8 \cdot 3$ & 9 & $10 \cdot 1$ \\
\hline $\begin{array}{l}\text { Serious injury, harm or death you } \\
\text { caused to someone }\end{array}$ & 33 & $6 \cdot 6$ & 10 & $8 \cdot 8$ & 23 & $6 \cdot 0$ & 4 & $4 \cdot 8$ & 23 & $7 \cdot 1$ & 6 & $6 \cdot 7$ \\
\hline
\end{tabular}

Table 2 Correlation between trauma exposure and eating disorder pathology, weight stigma, anxiety and specific eating disorder-associated behaviours and attitudes among a sample of clients presenting to food pantries affiliated with the San Antonio Food Bank, Texas, USA, JulyDecember 2016

\begin{tabular}{|c|c|c|c|c|c|c|c|c|}
\hline & EDDS & WSSQ & PSWQ & $\begin{array}{c}\text { Objective } \\
\text { binge eating }\end{array}$ & $\begin{array}{l}\text { Night } \\
\text { Eating }\end{array}$ & $\begin{array}{l}\text { Distress } \\
\text { after night } \\
\text { eating }\end{array}$ & $\begin{array}{c}\text { Self- } \\
\text { induced } \\
\text { vomiting }\end{array}$ & $\begin{array}{l}\text { Laxative/ } \\
\text { diuretic use }\end{array}$ \\
\hline Trauma exposure (LEC) & $-0.239^{\star \star \star}$ & $-0.151^{\star \star \star}$ & $-0 \cdot 210^{\star \star \star}$ & $-0.165^{\star \star \star}$ & $-0 \cdot 163^{\star \star \star}$ & $-0 \cdot 203^{\star \star \star}$ & $-0.115^{\star}$ & $-0.116^{\star}$ \\
\hline
\end{tabular}

EDDS, Eating Disorder Diagnostic Scale; WSSQ, Weight Self-Stigma Questionnaire; PSWQ, Penn State Worry Questionnaire; LEC, Life Events Checklist. Trauma exposure = score on LEC; lower scores $=$ more trauma exposure.

Objective binge eating = eaten an unusually large amount of food and felt loss of control; night eating = eaten an unusually large amount of food. Lower scores on LEC indicate higher levels of trauma exposure; for all other measures, higher scores indicate greater pathology.

${ }^{\star} P<0.05,{ }^{\star \star} P<0.01,{ }^{\star \star *} P<0.001$.

accident $\left(\chi_{(1, n 499)}^{2}=16 \cdot 33, P<0.001\right)$ and combat exposure $\left(\chi_{(1, n 499)}^{2}=25 \cdot 89, P<0.001\right)$, with male participants reporting elevated rates $(35.1$ and $16.7 \%$, respectively) relative to female participants $(17.4$ and $3.4 \%$, respectively). For ethnicity, we found a significant difference in sexual assault $\left(\chi_{(2, n 497)}^{2}=13 \cdot 75, P<0.001\right)$ and physical assault $\left(\chi_{(2, n 497)}^{2}=16 \cdot 95, P<0 \cdot 001\right)$, with participants in the 'Other' racial category reporting higher levels (31.5 and $40.4 \%$, respectively) compared with African-American participants (13.3 and $18.1 \%$, respectively) and Latino participants (15.4 and 20.6\%, respectively).

Consistent with our Aim 2 hypothesis, trauma exposure differed significantly between FI groups $\left(F_{(3,490)}=3 \cdot 35\right.$, $\left.P=0.019, \alpha^{2}=0.020\right)$. Post hoc tests indicated that the not food-insecure group (mean $=4.59, \mathrm{sD}=0.72)$ reported significantly less trauma exposure compared with the individual FI (mean $=4 \cdot 18, \mathrm{SD}=0.95)$ and child hunger FI groups $($ mean $=4 \cdot 16, \mathrm{SD}=0 \cdot 89)$. The household FI group $($ mean $=$ $4 \cdot 40, \mathrm{sD}=0.93)$ did not differ from any of the other groups.

Also consistent with our hypotheses, trauma exposure as indicated by LEC scores was significantly correlated with overall ED pathology, weight stigma and anxiety scores (see Table 2), with higher levels of trauma exposure being associated with increased psychopathology. In addition, LEC scores were significantly correlated with continuous measures of objective binge eating (i.e. eating an unusually large amount of food with a sense of loss of control), night eating (i.e. eating after waking up from sleep), frequency of distress after night eating, selfinduced vomiting to prevent weight gain or counteract the effects of eating, use of laxatives/diuretics to prevent weight gain or counteract the effects of eating, and weight/shape concerns. Note that all correlations are negative because lower scores on the LEC indicate higher levels of trauma exposure, as explained above.

\section{Discussion}

To our knowledge, the present four-aim study represents the first investigation of a wide range of potentially traumatic events in those living with FI in a high-income country. With regard to our first aim, to document rates of traumatic events in a low-income, marginalized and 
predominantly ethnic minority sample living with FI in the USA, results indicated that participants reported direct exposure to a wide range of potentially traumatic events as assessed by the LEC. In the present sample, $55.7 \%$ of the total sample reported direct exposure to a traumatic event and $61.6 \%$ reported direct exposure and/or witnessing such an event. Rates of reported traumatic event exposure likely vary with assessment method $^{(28)}$ and we were unable to find any civilian samples assessed with the LEC to compare with the present study. However, other studies using different methods (e.g. structured interview or the Traumatic Stress Schedule) have yielded similar trauma exposure rates in civilian samples. For instance, Norris ${ }^{(27)}$ found that $69 \%$ of a civilian sample reported lifetime exposure to at least one traumatic event. Kessler et ll $^{\left({ }^{(34)}\right.}$ found that $60.7 \%$ of men and $51.2 \%$ of women reported at least one potentially traumatic event in the National Comorbidity Study. Notably, a more recent study by Kilpatrick et al. ${ }^{(35)}$, using a national probability sample, found that $89 \%$ reported exposure to at least one DSM-5 traumatic event. Despite different populations and varied measures used, results for the present study (65.8\% for males; $53.2 \%$ for females) are similar to past research examining direct experience with a potentially traumatic event. Rates in the present study are a bit higher when witnessing is included $(71.1 \%$ for males; $59.5 \%$ for females).

Taken together, these results suggest that marginalized individuals with FI report frequent exposure to traumatic events at a rate similar to the general civilian population. On one hand, this might be viewed as good news; there are reasons to expect that an impoverished sample would experience higher rates of traumatic events than a general population sample as studies demonstrate that families living in urban poverty encounter multiple traumatic events and multifaceted risks associated with a lack of resources $^{(36)}$. On the other hand, those living with a constant lack of resources experience a chronic stressor, namely FI. Thus, FI might exacerbate symptomatology associated with traumatic event exposure to a greater degree compared with trauma's effect on more affluent populations; to our knowledge, no research has investigated this possibility. Further, those living with FI may have fewer resources to help manage the effects of a traumatic event (e.g. financial ability to seek psychological services, pay for medical expenses, meet financial obligations if temporarily disabled), which could exacerbate impact. For this reason, it will be important for future research to explore trauma-related symptomatology as trauma rates may be similar for the food-insecure and general populations, but post-traumatic stress disorder symptoms could differ.

With regard to specific types of traumatic events, participants in the present study reported a higher rate of physical and sexual assault than studies with similar overall rates of reported traumatic events. For instance, Norris $^{(27)}$ found that $15 \%$ of her total sample reported a lifetime history of physical assault; this contrasts with $23.7 \%$ in the present sample. Similarly, $4.4 \%$ of the Norris sample reported sexual assault, which contrasts with $17.9 \%$ in the current study. Given that women are more likely to report sexual assault, one possible reason for this difference could be the greater percentage of women in the present study compared with Norris. This explanation seems unlikely, however, given that rates of sexual assault and 'other unwanted sexual experiences' for both men and women (21.0 and 36.4\%, respectively) were higher in the present study than in Norris (lifetime frequency $1.3 \%$ for males and $7.3 \%$ for females). Similarly, Kessler et al. ${ }^{(34)}$ reported lower rates, with a combined total of $3.5 \%$ of males and $21.5 \%$ of females reporting they had experienced 'rape' or 'molestation'.

For physical assault, Kessler et al. ${ }^{(34)}$ reported $11 \cdot 1 \%$ for males and $6.9 \%$ for females; this contrasts with $25.4 \%$ of males and $23.4 \%$ of females in the present study. Norris did not appear to assess assault with a weapon, but a similar pattern emerges for this type of traumatic event when comparing Kessler et al.'s findings (19.0\% of males; $6.8 \%$ of females) with those of the present study (26.3\% of males; $14.0 \%$ of females). In contrast, combat exposure was similar for all three studies while fire-based traumatic events were nearly identical for Norris and the present study. In summary, results suggest that participants in the present study may be more prone to experiencing physical, sexual or non-combat weapon-based violence compared with a more nationally representative sample. These findings fit with a limited research literature exploring the intersection between violence and FI. These findings also support calls for increased focus on both research and conceptual frameworks to examine the relationship between violence and FI (e.g. Chilton and Booth ${ }^{(37)}$ ).

Regarding the second aim, consistent with our hypotheses, results indicate that participants in the child hunger and individual FI groups reported significantly greater trauma exposure than those in the not food-insecure group. The household FI group, which experiences anxiety about food running out before the end of the month, fell between these extremes but was not significantly different from any of the other FI groups. Because these data are cross-sectional, no inference can be drawn as to whether trauma exposure increases the likelihood that one becomes food-insecure, whether having limited income and suffering from FI increases trauma risk, whether low income increases risk for FI and trauma, or whether multidirectional relationships between these variables exist. Nevertheless, the current data highlight an important gap in the literature and suggest that additional research, preferably longitudinal, is needed to better understand the association of severity of FI with trauma exposure. Further research is also needed to explore the degree to which trauma exposure and its relationship with FI impact public health nutrition interventions aimed at those who are food-insecure. 
Results also indicate that within this food-insecure population, as hypothesized, greater trauma exposure was associated with increased ED symptomatology, internalized weight stigma and worry/anxiety. Trauma exposure also was significantly correlated with specific ED behaviours, including binge eating, night eating, selfinduced vomiting and laxative/diuretic use. Trauma has been long recognized as a non-specific risk factor for psychopathology ${ }^{(19,20)}$, so these results are not surprising. Despite this, the findings in the current study underscore the need to broaden the investigation of psychopathology and associated risk factors in those suffering with FI so that the full range of specific mental health problems associated with FI can be understood and potentially addressed.

More specifically, researchers need to explore how the chronic stressor of FI interacts with other risk factors (e.g. traumatic events) to untangle the varying associations with mental health status. For instance, it is possible that traumatic event severity and FI severity interact to create particularly pronounced problems in mental health. However, it is also possible that traumatic events increase psychopathology, which in turn leads to worsened FI.

Similarly, FI has been found to increase risk for obesity, which in turn increases the likelihood of both experienced and internalized weight stigma, each of which may worsen physical and mental health outcomes. Traumatic event exposure may interact in numerous ways in this sequence. For instance, research supports a link between early-life traumatic events (such as childhood physical or sexual assault) and adult obesity ${ }^{(38)}$. In addition, traumatic event exposure, particularly interpersonal trauma, such as physical or sexual assault, may increase an individual's awareness of and reaction to stigmatizing psychosocial messages, including those coming from well-meaning sources (e.g. anti-obesity campaigns that convey fat is bad).

ED pathology also could show similarly complex links. For instance, the dietary restraint associated with FI may directly increase risk for ED pathology (see Becker et $a l .{ }^{(14)}$ for additional discussion). However, the presence of traumatic events may further elevate risk $^{(19)}$. In addition, individuals who experience uncontrollable binge eating may worsen their ability to budget limited finances secondary to the cost of purchasing binge foods (http:// www.abcnews.go.com/Health/Diet/story?id=6256796\& page $=1$ ). Alternatively, food that was allocated to last over a set period of time may be uncontrollably consumed. Both scenarios could further worsen FI and the ability to feed one's family on a regular basis.

Developing a comprehensive understanding of the potentially complex links between psychopathology and FI is critical because individuals with FI who struggle with mental illness are less likely to benefit from programmes aimed at enhancing health status generally. For instance, diabetes is a major health concern for food-insecure populations in the USA and research indicates that depression is consistently associated both with decreased treatment adherence and worse treatment outcomes ${ }^{(39)}$. Moreover, by definition, ED are associated with dysregulation in eating, a key therapeutic target for most diabetes interventions. In summary, efforts to enhance the physical health of those with FI are likely to be impeded by unaddressed mental health problems.

Poor mental health also has been found to have a cyclical relationship with poverty, which in turn is a major risk factor for $\mathrm{FI}^{(40)}$. Research indicates that even in lowand middle-income countries, mental health interventions are associated with improved economic outcomes for those living in poverty ${ }^{(40)}$; moreover, improvements in economic outcomes have been shown to be linked to improvements in mental health status ${ }^{(40)}$. As such, it is imperative to better understand the complexity of mental health problems in those living with FI so that affordable and scalable interventions can be developed and implemented in low-income and racially diverse communities (see Collins et al. ${ }^{(36)}$ ).

The present study has a number of limitations. To start, because low levels of education are associated with poverty, we adjusted the wording and cut some items from several measures to make them comprehensible. This is a common approach when studying low-education and marginalized populations. However, this is less than optimal from a psychometric perspective. In addition, we did not conduct a validity study of the measures in Spanish, although we did back-translate all Spanish translations to make sure that the English and Spanish versions were equivalent. Fortunately, only a small number of participants completed the questionnaire in Spanish as over $75 \%$ of participants identified English as their first language.

As noted above, the present study is also limited because the data are cross-sectional. As such, no causal inferences can be drawn and longitudinal research will be needed to address questions of causation. When studying FI, it is important to understand its duration, intensity, and the impact FI has on individuals and families ${ }^{(36,41)}$. Therefore, both longitudinal and qualitative research will be necessary to replicate and extend the present findings, particularly as they relate to chronic FI, transitory FI and seasonal $\mathrm{FI}^{(40)}$. Longitudinal research will also provide information to determine if ED precede or result from FI. This knowledge can influence future programmes designed to assist food-insecure populations.

An additional limitation comes from our use of the LEC as an indicator of traumatic event exposure. Although the LEC has good psychometric properties and largely addressed the aims of the present study, it is limited in that it does not assess the volume or severity of exposure to each type of traumatic event. As such, all direct experience is essentially equated. Future research needs to utilize other methods to assess trauma in addition to the LEC. 
We used a convenience sample based on who visited a specific food pantry on a day we were collecting data and if an adult was willing to participate; there were no additional requirements of participants. Despite using a convenience sample, it was a purposive sample, which was advantageous to the study. We wanted participants who were representative of low-income and food-insecure individuals and families in San Antonio to begin to answer research questions that focused on one particular trait of interest, FI. Another strength of the present study is the assessment of the level of FI. Instead of assuming all individuals who seek food from a food pantry are equally food-insecure, we differentiated between the levels of FI experienced by participants in our sample.

\section{Conclusion}

In conclusion, the present study underscores the need to conduct more research to understand the full range of mental health problems and traumatic events in those with FI and to explore these relationships in the context of nutrition-based public health initiatives. Further, studies should also focus on explicating the relationship of trauma to mental health in those living with the chronic stressor of FI. More specifically, future research should investigate the degree to which FI and a history of trauma might interact to produce particularly elevated levels of psychopathology. This research will likely provide critical information to supplement public health efforts designed to address mental and physical health concerns in food-insecure populations.

\section{Acknowledgements}

Acknowledgements: The authors would like to thank the San Antonio Food Bank and affiliated Food Pantries for their assistance in this research. Financial support: This work was supported by HEB Grocery Company, McNair Scholars Program and Murchison Summer Undergraduate Research Fellowship Program. The funders had no role in the design, analysis or writing of this article. Conflict of interest: None. Authorship: C.B.B. contributed to the design and implementation of the study, data analysis, and the conceptualization and writing of this paper. K.M. contributed to the design and implementation of the study, and the conceptualization and writing of this paper. C.J. and B.T. contributed to the design and implementation of the study and data entry. F.G. contributed to the implementation of the study, data entry, cleaning of data, data analysis, literature review and writing. A.S. contributed to the cleaning of data, data analysis, literature review and writing. All authors reviewed and approved the final version. Ethics of buman subject participation. This study was conducted according to the guidelines laid down in the Declaration of Helsinki and all procedures involving human subjects were approved by the Trinity University Institutional Review Board. Written informed consent was obtained from all participants.

\section{References}

1. Lang T \& Barling D (2012) Food security and food sustainability: reformulating the debate. Geogr J 178, 313-326.

2. Food and Agriculture Organization of the United Nations (2008) An Introduction to the Basic Concepts of Food Security. http://www.fao.org/docrep/013/al936e/al936e00.pdf (accessed September 2017).

3. Coleman-Jensen A, Rabbitt M, Gregory C et al.2016) Household Food Security in the United States in 2015. Economic Research Report no. ERR-215. Washington, DC: US Department of Agriculture, Economic Research Service.

4. McIntyre L, Glanville NT, Raine KD et al. (2003) Do lowincome lone mothers compromise their nutrition to feed their children? CMAJ 168, 686-691.

5. Gucciardi E, Vahabi M, Norris N et al. (2014) The intersection between food insecurity and diabetes: a review. Curr Nutr Rep 3, 324-332.

6. Seligman HK, Laraia BA \& Kushel MB (2009) Food insecurity is associated with chronic disease among low-income NHANES participants. J Nutr 140, 304-310.

7. Vozoris NT \& Tarasuk VS (2003) Household food insufficiency is associated with poorer health. J Nutr 133, 120-126.

8. Leung CW, Epel ES, Ritchie LD et al. (2014) Food insecurity is inversely associated with diet quality of lowerincome adults. $J$ Acad Nutr Diet 114, 1943-1953.

9. Alaimo K, Olson CM \& Frongillo EA (2002) Family food insufficiency, but not low family income, is positively associated with dysthymia and suicide symptoms in adolescents. J Nutr 132, 719-725.

10. Davison KM \& Kaplan BJ (2015) Food insecurity in adults with mood disorders: prevalence estimates and associations with nutritional and psychological health. Ann Gen Psychiatr 14, 21.

11. Muldoon KA, Duff PK, Fielden S et al. (2013) Food insufficiency is associated with psychiatric morbidity in a nationally representative study of mental illness among food insecure Canadians. Soc Psychiatry Psychiatr Epidemiol 48, 795-803.

12. Palar K, Kushel M, Frongillo EA et al. (2015) Food insecurity is longitudinally associated with depressive symptoms among homeless and marginally-housed individuals living with HIV. AIDS Behav 19, 1527-1534.

13. Sorsdahl K, Slopen N, Siefert K et al. (2010) Household food insufficiency and mental health in South Africa. J Epidemiol Community Health 5, 426-431.

14. Becker CB, Middlemass K, Taylor B et al. (2017) Food insecurity and eating disorder pathology. Int J Eat Disord 50, 1031-1040.

15. Hudson JI, Hiripi E, Pope HG et al. (2007) The prevalence and correlates of eating disorders in the National Comorbidity Survey Replication. Biol Psychiatr 61, 348-358.

16. Carta MG, Preti A \& Moro MF (2014) Eating disorders as a public health issue: prevalence and attributable impairment of quality of life in an Italian community sample. Int Rev Psychiatry 26, 486-492.

17. Pearl RL, Wadden TA \& Hopkins CM (2017) Association between weight bias internalization and metabolic syndrome among treatment-seeking individuals with obesity. Obesity (Silver Spring) 25, 317-322.

18. Melchior M, Caspi A, Howard LM et al. (2009) Mental health context of food insecurity: a representative cohort of families with young children. Pediatrics 124, 564-572. 
19. Brewerton TD (2007) Eating disorders, trauma, and comorbidity: focus on PTSD. Eat Disord 15, 285-304.

20. Turner RJ \& Lloyd DA (1995) Lifetime traumas and mental health: the significance of cumulative adversity. J Health Soc Behav 1, 360-376.

21. Chilton MM, Rabinowich JR \& Woolf NH (2014) Very low food security in the USA is linked with exposure to violence. Public Health Nutr 17, 73-82.

22. Wehler C, Weinreb LF \& Huntington N (2004) Risk and protective factors for adult and child hunger among low-income housed and homeless female-headed families. Am J Public Health 94, 109-115.

23. Roberts B, Ocaka KF, Browne J et al. (2009) Factors associated with the health status of internally displaced persons in northern Uganda. J Epidemiol Community Health 63, 227-232.

24. Kendall A, Olson CM \& Frongillo EA (1995) Validation of the Radimer/Cornell measures of hunger and food insecurity. J Nutr 125, 2793-2801.

25. Radimer KL, Olson CM, Greene JC et al. (1992) Understanding hunger and developing indicators to assess it in women and children. J Nutr Educ 24, Suppl. 1, 36S-44S.

26. Gray MJ, Litz BT, Hsu JL et al. (2004) Psychometric properties of the life events checklist. Assessment 11, 330-341.

27. Norris FH (1992) Epidemiology of trauma: frequency and impact of different potentially traumatic events on different demographic groups. J Consult Clinical Psychol 60, 409.

28. Norris FH \& Slone LB (2013) Understanding research on the epidemiology of trauma and PTSD. PTSD Res $Q \mathbf{2 4}$, 1-5; available at https://www.ptsd.va.gov/professional/ newsletters/research-quarterly/v24n2-3.pdf

29. Stice E, Fisher M \& Martinez E (2004) Eating disorder diagnostic scale: additional evidence of reliability and validity. Psychol Assess 16, 60-71.

30. Krabbenborg MA, Danner UN \& Larsen JK (2012) The eating disorder diagnostic scale: psychometric features within a clinical population and a cut-off point to differentiate clinical patients from healthy controls. Eur Eat Disord Rev 20, 315-320.
31. Lillis J, Luoma JB, Levin ME et al. (2010) Measuring weight self-stigma: the weight self-stigma questionnaire. Obesity (Silver Spring) 18, 971-976.

32. Meyer TJ, Miller ML, Metzger RL et al. (1990) Development and validation of the Penn State worry questionnaire. Behav Res Ther 28, 487-495.

33. Fiske L, Fallon EA, Blissmer B et al. (2014) Prevalence of body dissatisfaction among United States adults: review and recommendations for future research. Eat Behav 15, 357-365.

34. Kessler RC, Sonnega A, Bromet E et al. (1995) Posttraumatic stress disorder in the National Comorbidity Survey. Arch Gen Psychiatry 52, 1048-1060.

35. Kilpatrick DG, Resnick HS \& Milanak ME (2013) National estimates of exposure to traumatic events and PTSD prevalence using DSM-IV and DSM-5 criteria. J Trauma Stress 26, 537-547.

36. Collins K, Connors K, Davis S et al. (2010) Understanding the Impact of Trauma and Urban Poverty on Family Systems: Risks, Resilience, and Interventions. Baltimore, MD: Family Informed Trauma Treatment Center.

37. Chilton M \& Booth S (2007) Hunger of the body and hunger of the mind: African American women's perceptions of food insecurity, health and violence. J Nutr Educ Behav 39, 116-125.

38. D'Argenio A, Mazzi C, Pecchioli L et al. (2009) Early trauma and adult obesity: Is psychological dysfunction the mediating mechanism? Physiol Behav 98, 543-546.

39. Gonzalez JS, Peyrot M, McCarl LA et al. (2008) Depression and diabetes treatment nonadherence: a meta-analysis. Diabetes Care 31, 2398-23403.

40. Lund C, De Silva M, Plagerson S et al. (2011) Poverty and mental disorders: breaking the cycle in low-income and middle-income countries. Lancet 378, 1502-1514.

41. Food and Agriculture Organization of the United Nations (2009) The State of Food Insecurity in the World 2009. Meeting the 2015 international hunger targets: taking stock of uneven progress. ftp://ftp.fao.org/docrep/fao/012/i0876e/ i0876e_flyer.pdf (accessed September 2017). 